\title{
Ion spectroscopy - A diamond characterization tool
}

\author{
E. Berdermann ${ }^{\text {a,* }}$, A. Caragheorgheopol ${ }^{\text {b }}$, M. Ciobanu ${ }^{\text {a }}$, M. Pomorski ${ }^{\text {a }}$, A. Pullia ${ }^{\text {c }}$, S. Riboldi ${ }^{\text {, }}$ \\ M. Traeger ${ }^{a}, H$. Weick ${ }^{a}$ \\ a Gesellschaft für Schwerionenforschung mbH Darmstadt, Germany \\ ${ }^{\mathrm{b}}$ National Institute for Physics and Nuclear Engineering, Romania \\ c INFN and University of Milan, Italy
}

\section{A R T I C LE I N F O}

Available online 4 April 2008

\section{Keywords:}

CVD-diamond

Heavy-ion detectors

Energy-loss spectroscopy

Diamond characterization

\begin{abstract}
A B S T R A C T
The stopping power $\Delta E$ and the energy-loss resolution $\delta E / \Delta E$ of Single-Crystal CVD-Diamond Detectors (SC-DDs) measured with relativistic Heavy Ions (HIs) are interpreted as global quality parameters, characterizing simultaneously crystal texture and carrier-trapping concentrations, as well as the chargetransport properties of intrinsic diamond samples. HI spectra are presented, where spectral lines are obtained similar to the predictions of the Lindhard and Sørensen (LS) theory [J. Lindhard, A.H. Sørensen, Phys. Rev. A 53 (1996) 2443]. The spectroscopic results indicate an almost defect free material, and spatial homogeneity of all parameters relevant to the detector signal (i.e., mass density, dielectric constant, drift mobility and velocity of the charge carriers). Measured and simulated transient current signals generated by relativistic ${ }^{132}$ Xe ions are discussed according to theories [A. Many, G. Rakavy, Phys. Rev., 126 (1962) 1980; G. Juška, M. Viliunas, O. Klíma, E. Šípek, J. Kočka, Phil. Mag. B 69 (1994) 277; G. Juška, M. Viliunas, K. Arlauskas, J. Kočka, Phys. Rev. B 51 (1994) 16 668] of space-charge limited current (SCLC) transients. The evidence of the spectroscopic results is confirmed by the current-mode studies, and thus indirectly, the potential of the characterization method as well.
\end{abstract}

(C) 2008 Elsevier B.V. All rights reserved.

\section{Introduction}

The knowledge of the interaction of HIs slowing down in diamond is important for a variety of new detector applications, ranging from nuclear physics experiments and nuclear waste investigations up to medical applications in tumour therapy with carbon ions and protons [5-7]. Aiming for precise measurements of the so far missing stopping power of diamond and the corresponding energy-loss of heavy charged particles in it, an electrical characterization method for highquality materials is found, which completes the measurements performed with short-range ionizing sources (e.g., $\alpha$-particles [8-10], UV photons [11]) or with minimum ionizing particles (MIP) [12]). Even if MIPs show similarities to the probes proposed in this article, they generate in contrast weak charge signals and spectra revealing the characteristic asymmetric shape of a Landau distribution [13].

Relativistic ions provide a homogeneous ionization profile and enable characterization of dual-carrier drift in a moderate spacecharge limited regime. They traverse a few hundred micrometers material in a short time ( $\sim 1 \mathrm{ps})$, while depositing the same amount of energy per unit path length through the whole sample thickness. A charge $Q_{G}$ is generated instantaneously $(t=0)$ along the particles path, creating a non-equilibrium condition in the sensor which is char-

\footnotetext{
* Corresponding author.

E-mail address: e.berdermann@gsi.de (E. Berdermann).
}

acteristic for both, the detector material and the traversing ion of $Z_{\mathrm{eff}}$, $A, \beta$ (with $Z_{\text {eff }}$ the ionic charge state, $A$ the mass and $\beta$ the velocity of the particle). In the data discussion, the experimental results will be compared to values calculated according to LS [1], which has overruled the Bethe-Bloch theory in the relativistic ion range.

In the configuration of an ion spectroscopy detector, a sample is biased with $U_{\text {Bias }}$ and read out by an integrator circuit of a decay time constant $\mathrm{RC}>t_{\mathrm{Tr}}$, the latter being the thickness dependent transition time of the collected charge which is for intrinsic mono-crystalline CVD diamond in the order of a few nanoseconds [10]. The separated electrons and holes drift to both electrodes (dual-carrier current), while creating an 'ion-induced' current signal. After the last moving carriers arrived at the electrodes, the ion-induced current is fully integrated at the circuit capacitance $C$, and the amplitude of the resulting voltage pulse on the output of a charge-sensitive spectroscopy amplifier corresponds to the collected charge $Q_{c}$. If the charge transport was perfect, the collected charge is equal to the generated charge $Q_{G}$ at all times, and the best possible energy resolution is achieved.

At relativistic velocities, where even heaviest ions are fully ionized, $Z_{\text {eff }}$ becomes the nuclear charge $Z$ of the particle and the interpretation of the measured spectral lines is simple. Moreover, in dense and thick absorbers, the energy-loss distributions are Gaussians [14]. We analyze therefore the most probable value being equal to the mean energy loss $\Delta E$, and the Full Width at Half Maximum (FWHM) of the lines which corresponds to the energy-loss straggling $\delta E$. 
The total experimental width is given by Eq. (1)

$\delta E_{\mathrm{Sum}}=\sqrt{\delta E_{\mathrm{G}}^{2}+\delta E^{2}+\delta E_{\mathrm{Dia}}^{2}+\delta E_{\mathrm{Noise}}^{2}}$

with $\delta E_{\mathrm{G}}$ the energy uncertainty introduced by statistical fluctuations of charge generation processes, $\delta E$ the energy-loss straggling caused by different impact parameters (i.e. roughly, the distance of closest approach of the incident particle to the centre of a diamond atom), $\delta E_{\text {Dia }}$ the trapping and recombination related diamond term, and $\delta E_{\text {Noise }}$ the electronic noise contribution.

Negligible influence is due to the charge generation uncertainty, since $\delta E_{\mathrm{G}}$ obeys the Eq. (2)

$\delta E_{\mathrm{G}}=\sqrt{F_{\mathrm{Dia}} \cdot \varepsilon_{\mathrm{Dia}} \cdot \Delta E}$

with $F_{\text {Dia }}$ the diamond Fano factor [15], $\varepsilon_{\text {Dia }}$ the average energy required to produce an e-h pair in diamond, and $\Delta E$ the energy loss of the traversing ion.

The pair-production energy $\varepsilon_{\text {Dia }}$ is measured for SC-D material from Ref. [16] to $(12.84 \pm 0.03) \mathrm{eV} / \mathrm{e}-\mathrm{h}$ [9]. No reliable measurements exist so far for $F_{\text {Dia. }}$. However, assuming a typical value of crystal detectors (e.g. $F_{\mathrm{Dia}} \sim 0.1-0.2$ ) the charge generation is not limiting the energy resolution of $\mathrm{HI}$ detectors. For instance, even for $\Delta E \sim 1 \mathrm{GeV}-$ which is the case of the measurements described in Section $3-$ the ratio $\delta E_{\mathrm{G}} / \Delta E$ is $\sim 5 \times 10^{-5}$. The diamond energy-loss resolution $\delta E_{\mathrm{Dia}} /$ $\Delta E_{\text {Dia }}$, where $\Delta E_{\text {Dia }}$ is the measured peak value of an ion distribution, is extracted from Eq. (1) considering the calculated straggling $\delta E$ according to LS and by measuring $\delta E_{\text {Noise }}$.

We report on first characterization measurements with relativistic ions performed at the high-resolution spectrometer FRagment Separator (FRS) of GSI [17] (Section 2). SC-DDs and a silicon pin-diode detector were tested with heavy particles produced by ${ }^{132} \mathrm{Xe}$ fragmentation on a $4 \mathrm{~g} / \mathrm{cm}^{2}$ beryllium target (Section 3). Original transient current (TC) signals, generated by primary ${ }^{132} \mathrm{Xe}$ projectiles of $215 \mathrm{MeV} / \mathrm{amu}$ kinetic energy in SC-DDs readout in 'current mode', are discussed in Section 4. An attempt is made to compare the experimental transients with theoretical SCLC data [2-4] as well as with simulated signals.

For simplicity and beam-time reduction, projectile fragments have been used in this study. However, it is of high scientific interest to compare measured diamond stopping powers with tabulated standard carbon data, extrapolated so far for the estimation of theoretical diamond values. It is intended to test the validity of the density-effect correction for the tight-coupling case of carbon atoms in the diamond lattice and to measure reliably the diamond Fano factor [16], giving inside view to phonon creation and propagation, to the dielectric constant $\varepsilon(\omega)$, and more. Therefore, high-precision measurements will be performed in a future experiment with individual ion beams having the samples placed in vacuum at the focal plane F2 of the FRS (Fig. 1), and the magnetic system operating in the energy-loss spectrometer mode [17]. In addition, the SCLC transients will be recorded with a remote controlled $15 \mathrm{GHz}$ DSO of $45 \mathrm{GS} / \mathrm{s}$ resolution, minimizing the influence of the electronic bandwidth to peculiarities of the TC signals. Such precision data are only enabled by high-quality SC-D samples, as supplied by [16].

\section{Experimental details}

We tested two intrinsic SC-D samples (D1, D2) of $4 \mathrm{~mm} \times 4 \mathrm{~mm}$ area and of $400 \mu \mathrm{m}$ thickness along with a silicon pin-diode detector (Si1) of same thickness and similar size. The sensors were mounted in air on a remote-control platform movable perpendicular to the beam direction. The same integrating amplifier was used for all measurements. It was equipped with an internal bias line connecting the beam-side electrode to the high pole of a power supply. The rear side electrode (at zero potential) was connected to the (shielding) metallic detector box.

Fig. 1 shows on top a schematic of the FRS spectrometer with its four sections S1 to S4, and the corresponding focal planes F1 to F4. The target area is indicated on the left side of the picture (TA). A diamond under test and the diagnosis detectors used in these measurements are sketched in a zoomed scale below the magnets. The beam quality was optimized by several slits and the beam position along the $70 \mathrm{~m}$ beam lines of the FRS was displayed using Multi-Wire-Proportional Counters (MWPC) of a spatial resolution $\leq 1 \mathrm{~mm}$. Even for primary xenon ions the beam spots on the diamonds were larger than the active detector areas, enabling some background due to border events of different timing and shape [18]. In order to ensure that measured diamond signals were only related to atomic interactions of individual incident ions, an independent Particle IDentification (PID) was performed by coincident measurements of $\Delta E$ spectra with two highresolution gaseous Multiple-Sampling Ionization Chambers (MUSIC), one placed in front (MUSIC41) and the second after the test detectors (MUSIC42). In an event-by-event basis, MUSIC signals were corrected online, taken into account the Time-of-Flight (ToF) of the ions measured between F2 and F4 (distance F2-F4=36 m) with plastic scintillator detectors. Hence, isotopes of same magnetic rigidity $B \rho$ are ejected in the MUSIC PID spectra. Note that this sensor type represents the state-of-the-art detectors for $\mathrm{HI}$ fragmentation

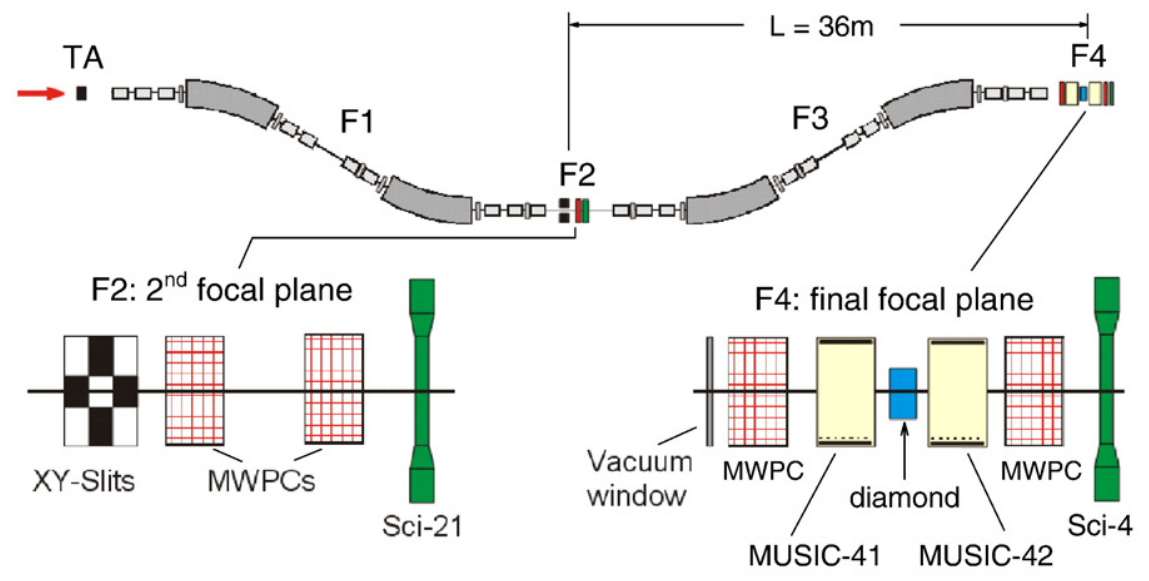

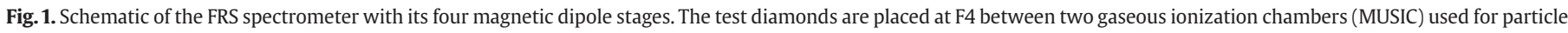

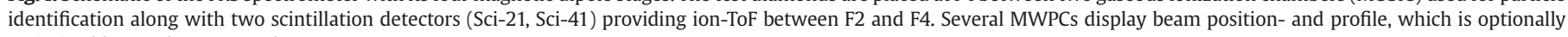
optimized by XY-slits mounted at F2. 

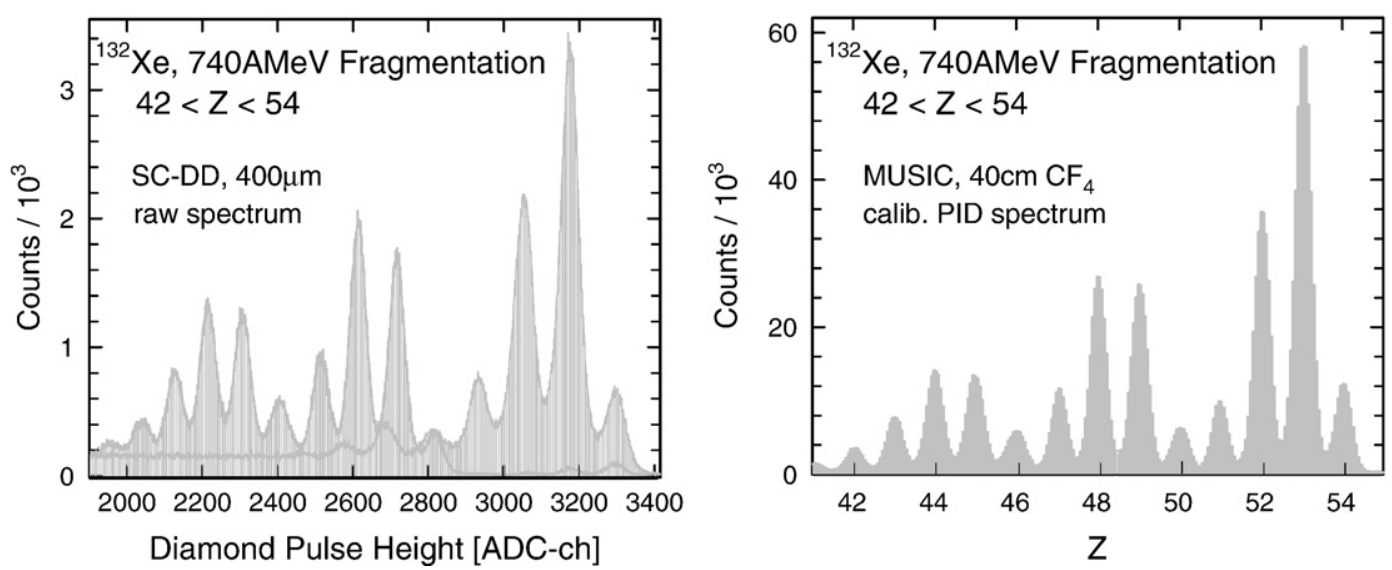

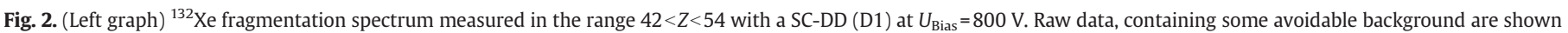
(see text). (Right graph) The corresponding Ion-ToF corrected MUSIC41 PID spectrum, where isotopes of same rigidity are excluded.

spectroscopy [19]. Consisting of 8 anode strips, multiple $\Delta E$ measurements are performed within an active length of $40 \mathrm{~cm}$ CF4 gas.

\section{Heavy-ion spectra measured with SC-DD and silicon detectors}

Particles, of different $Z$ and $A$ created by fragmentation of ${ }^{132} \mathrm{Xe}$ ions of $740 \mathrm{MeV} / \mathrm{amu}$ kinetic energy on a beryllium target, were bend by the magnetic system of the FRS to hit almost perpendicularly the test sensors at F4. Fig. 2 shows on the left graph pulse-height spectra measured with D1 at $800 \mathrm{~V}$ (raw data) and on the right graph the corresponding MUSIC PID distributions. The data are obtained with three different FRS settings of individual transmission profiles chosen to cover consecutive $Z$ regions. The variation of the line intensities is due to the transport function of the FRS for a given setting of the magnets. In reality, the fragmentation cross-section decreases exponentially with $Z$.

Diamond PID spectra are not available at the present status of data analysis, and consequently, the SC-DD spectrum includes some contamination by isotopes of same rigidity, which are eliminated in the MUSIC PID distribution. Considering this avoidable background as well as the well-understood effect of border events [18], a similar resolution of both sensors is concluded.

In Fig. 3 (left graph), energy-calibrated D1- and D2 pulse-height distributions measured in the range $45<Z<54$ (black lines) are presented along with the Si1 spectrum (grey line) obtained in the range $50<Z<54$. The superior performance of diamond in this experiment

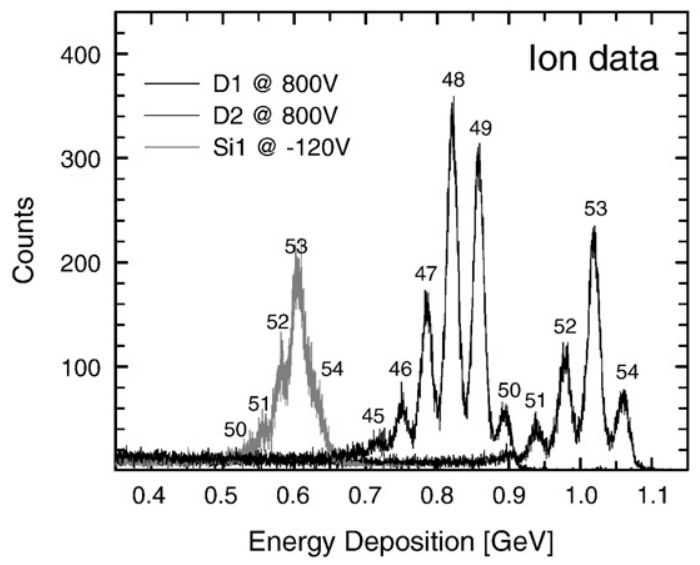

is evident, although before the HI run Si1 showed slightly better

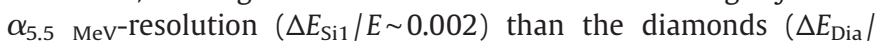
$E \sim 0.003$ ) [9]. Almost complete charge collection leading to a particle identification power $\Delta Z_{\text {Dia }} / Z<1$ and to an energy resolution $\delta E_{\text {Dia }} /$ $\Delta E_{\text {Dia }} \sim 2.5 \%$ is obtained for both diamond detectors, whereas for Si1, pulse-height defects in the order of $5.5 \%$ are observed. Due to that, we measured for silicon $\Delta Z_{\mathrm{Si} 1} / Z>1$ and an energy resolution $\delta E_{\mathrm{Si} 1} /$ $\Delta E_{\text {Si1 }}>4.6 \%$, respectively.

The Si1 result cannot be explained by intense beams or radiation damage. In spectroscopic measurements the rates are low $(\leq 1 \mathrm{kHz})$ and the total estimated fluence on $\mathrm{Si} 1$ was $\sim 10^{5} \mathrm{p} / \mathrm{cm}^{2}$, i.e. much lower than the integral rate on D1 and D2. We correlate the deterioration of Si1 with the lower pair-production energy of the material $\left(\varepsilon_{\mathrm{Si}}=3.61 \mathrm{eV} / \mathrm{e}-\mathrm{h} \sim 1 / 4 \varepsilon_{\text {Dia }}\right)$, which results to background sensitivity and higher ionization density in silicon sensors, though the ion energy loss is smaller than in diamond (Fig. 3, left graph). In addition, the slow hole-drift in silicon hinders a fast absorption of the transient space charge. The charge collected from both detector types is plotted on the right graph of Fig. 3 with the pulse heights calibrated in a material independent value $(\mathrm{pC})$.

The peak values measured with D1 and D2 are plotted in Fig. 4 (left graph) as a function of $Z^{2} / \beta^{2}$. The experimental data (black dots) are in good agreement with the theoretical calculations (open circles), demonstrating charge-collection efficiency close to ideal, and thus, negligible trapping and recombination. The latter is confirmed by the relative widths, plotted on the right graph (black dots) versus $Z$, which

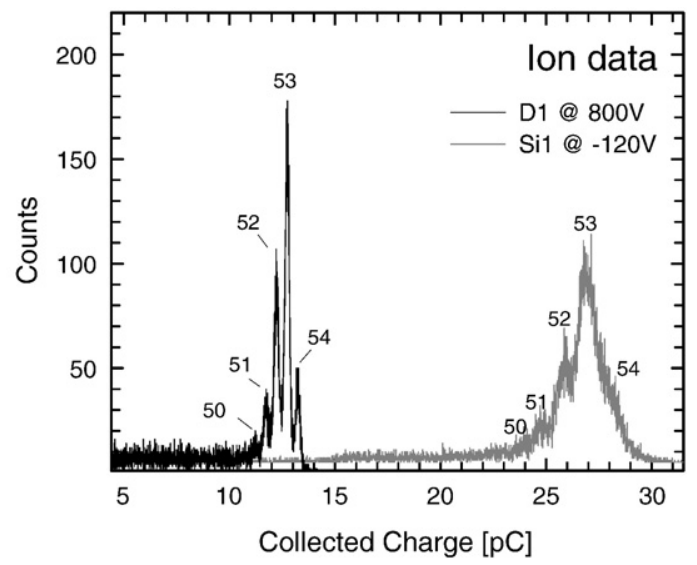

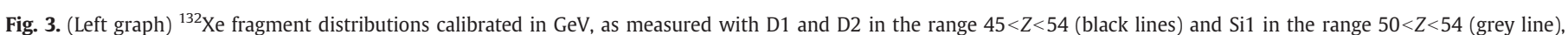

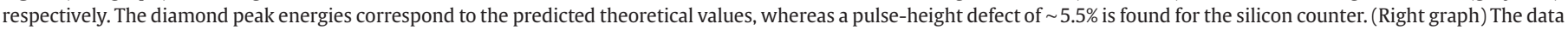

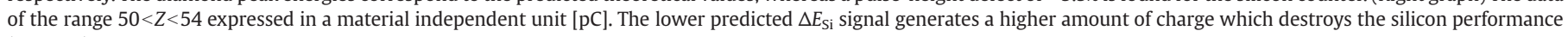
(see text). 

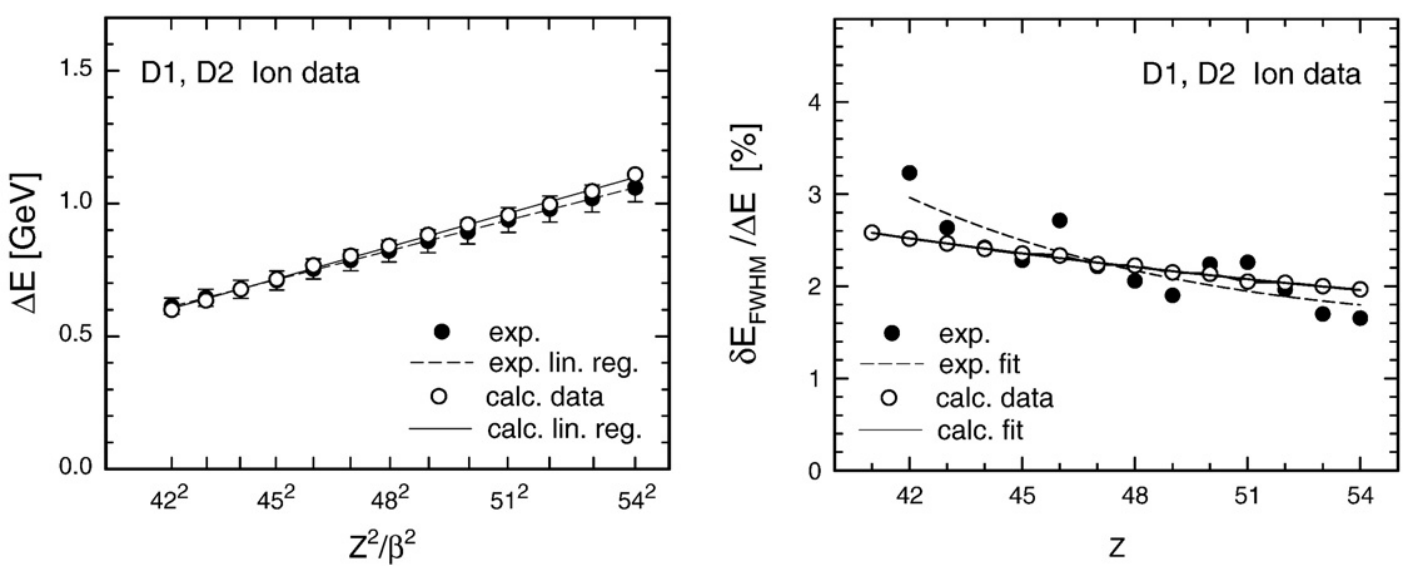

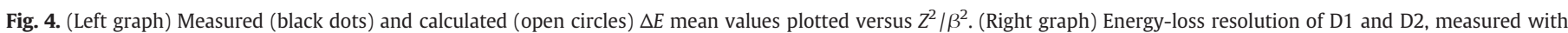

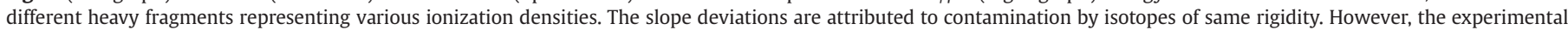
data are close to the predicted theoretical values.

are in the same order of the theoretical energy-loss straggling (open circles). In both plots the experimental data show slightly different slopes compared to theory, which are attributed to a weak line contamination by isotopes of same rigidity and to border events [18]. However, the descending trend of the $Z$ dependence indicates not only the trivial fact of increasing $\mathrm{S} / \mathrm{N}$ ratio but it demonstrates also the absence of space-charge accumulation and a high mobility of electrons and holes providing quick restoration of the electric field. The data suggest in addition that high-energy $\delta$-rays (knock-on electrons) escape from the active detector volume, as it is also the case for the high-resolution gaseous MUSIC chambers. This is a desirable property of sensor materials used for ion spectroscopy, since $\delta$-rays contribute strongly to the energyloss straggling and very little to the mean energy loss [14].

\section{Transient current signals}

Original (non-amplified) TC signals created by ${ }^{132} \mathrm{Xe}$ ions of $215 \mathrm{MeV} / \mathrm{amu}$ kinetic energy have been analyzed with a $1 \mathrm{GHz}$ DSO of $10 \mathrm{GS} / \mathrm{s}$ resolution. The RC time constant of the system was in this case as short as $45 \mathrm{ps}$ at $50 \Omega$ impedance, i.e. $\mathrm{RC}<t_{\mathrm{Tr}}$ [10]. The bias resistor was chosen to $10 \mathrm{k} \Omega$, in order to ensure constant voltage on the electrodes at all times, and thus to perform the measurements in the so called 'current mode'. In the left graph of Fig. 5, we present the ion-induced current transients obtained with the beam-side electrode at positive bias. The ion-induced charge $Q_{c}$ corresponds to the integrated area of the single-particle TC signals. It has been demonstrated in detail [18] that $Q_{c}$ saturates to the expected theoretical level at $U_{\text {Bias }} \sim 40 \mathrm{~V}$.

The system relaxes back to equilibrium in a time $t_{\mathrm{R}}>t_{\mathrm{Tr}}$, which is the time needed to expel all excess space-charge generated in the crystal [2]. Immobile minority carriers or a high intrinsic carrier density affect the relaxation time decreasing the internal electric field, and thus, $t_{\mathrm{R}}$ is shorter for low-quality DDs (e.g., PC diamond sensors [20]). Charge dispersion has minor effect on $t_{R}$, but it causes a round off of the signal drop after charge extraction [2].

The bias induced charge $Q_{\mathrm{Bi}}=C_{\mathrm{det}} * U_{\text {Bias }}$ on the electrodes was changed from $9 \mathrm{pC}$ for $10 \mathrm{~V}$ to $720 \mathrm{pC}$ for $800 \mathrm{~V}$, respectively, by increasing $U_{\text {Bias }}$ at constant ion-generated charge $Q_{\mathrm{G}}=19.22 \mathrm{pC}$. The plot parameter on the left graph of Fig. 5 is expressed in units of $Q^{\prime}=$ $Q_{B i} / Q_{G}$. It is necessary to notice that the low bandwidth of the available DSO may smear out some peculiarities of the recorded transients.

The interpretation of the measured TC signals according to $[3,4]$ is limited by several assumptions non-applicable to our experiment. Instead of a homogeneous ionization profile through the whole diamond bulk, as provided by relativistic HIs, strongly absorbed UV light is used as injecting source, which decreases exponentially behind the
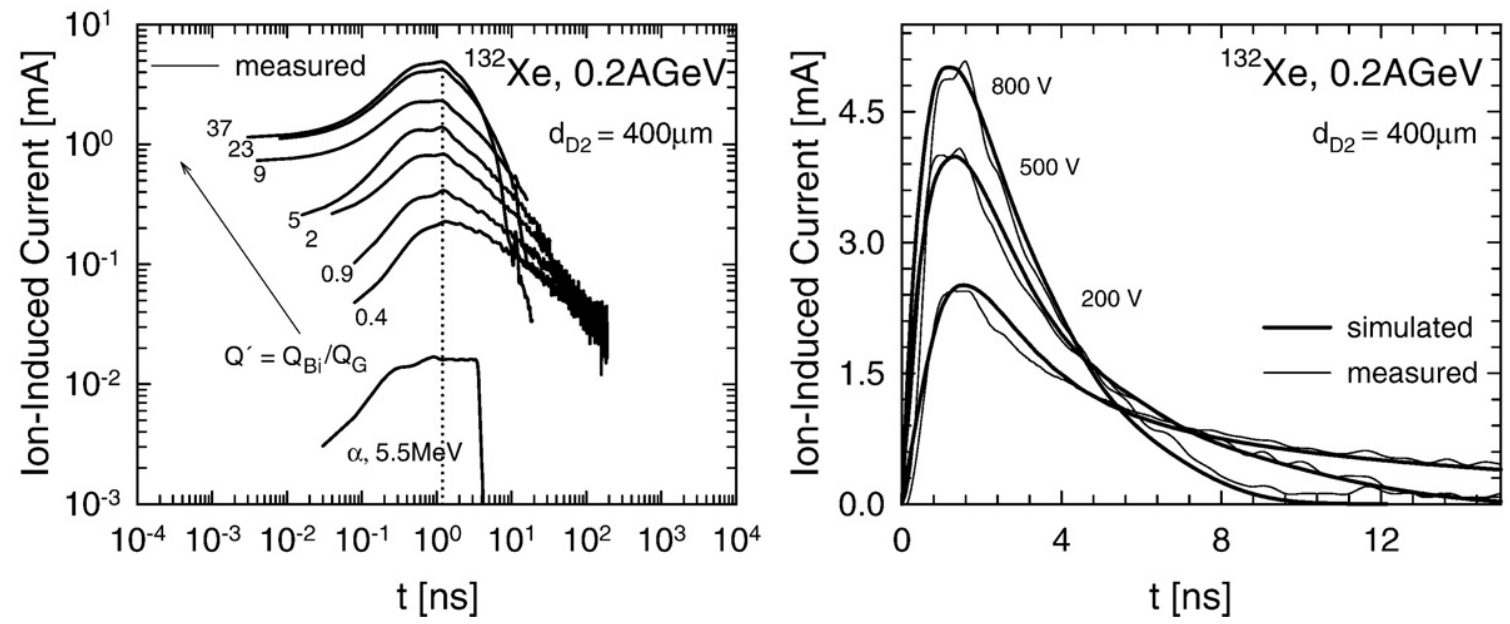

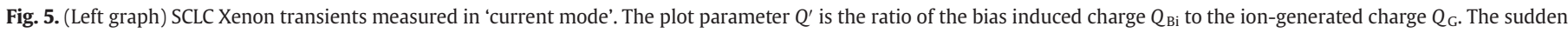

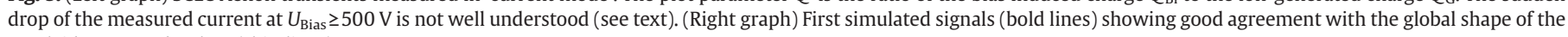
overlaid measured pulses (thin lines). 
blocking contacts. Furthermore, the theory [2-4] refers usually to pindiode structures, where single-carrier drift is assumed. If the case of dual-carrier drift is treated [3], significantly lower mobility of minority carriers is implemented, which is not correct for diamond [10]. It is also not clear if the variation of $Q_{\mathrm{Bi}}$ at constant $Q_{\mathrm{G}}$, as performed in the present study, is compatible to the variation of the charge injection $Q_{C}$ at constant bias.

However, an attempt is made to extract some analogue features. A continuous shift of the 'cusp', which would indicate a decrease of the effective sample thickness with decreasing $Q^{\prime}$ [3], is not supported for the case of increasing $Q_{\mathrm{Bi}}$ (see dotted line at constant $t$-cusp), at least not in these measurements. Representing the single-carrier drift in the low-signal case, a ${ }^{241} \mathrm{Am}-\alpha$-signal (obtained at $400 \mathrm{~V}$ ) is plotted in addition. Note that the range of $5.5 \mathrm{MeV} \alpha$-particles in diamond is $\sim 12 \mu \mathrm{m}$ and the corresponding $\alpha$-generated charge $Q_{\mathrm{G}}=68 \mathrm{fC}$.

Both kinks of the 'cusp' reported in [3] are visible but not pronounced, and in particular, no decreasing slope of the TC is observed for $t<t_{T r}$, as it is the case of different mobilities of majority and minority carriers (or bulk trapping) in the dual-drift mode [3]. Whenever the kinks appear clearly (for $U_{\text {Bias }} \geq 500 \mathrm{~V}$ ), the later one is more intense. This may indicate a 'leakage' of the anode at high electric fields, leading to electron injection and accumulation of a weak charge reservoir, which is dropped out at a time corresponding to the "third kink' discovered by [3]. However, the collection efficiency amounting correctly to $\sim 100 \%$ for this bias range [18], is in contradiction to this assumption. Since the influence of contacts is still not clearly understood, the origin of the sudden drop down of the ion-induced current cannot be reliably concluded.

The right graph of Fig. 5 shows measured SCLC transients at $E \geq 0.5 \mathrm{~V} / \mu \mathrm{m}$ (thin lines) compared with simulated signals (bold lines). At the current status of the simulations, the following input parameters are implemented: no recombination (infinite carrier lifetime), a diamond bulk almost without impurities (number of acceptors $N_{\mathrm{A}}=10^{3}$ ), intrinsic carrier concentration $N_{\text {intr }}=10^{5} / \mathrm{cm}^{3}$, and a fielddependent mobility. The initial transport parameters are taken from $\alpha$-measurements reported in [10]. Corresponding to the generated charge $Q_{\mathrm{G}}=19.22 \mathrm{pC}$, a total number of $1.2 * 10^{8} \mathrm{e}$ is injected homogeneously in ten points along the beam axis of a diamond material of relative permittivity $\varepsilon_{\mathrm{r}}=5$.7. The simulated signals are in good agreement with strength and global shape of the measured transients, but do not describe well the peculiarities of the cusp. More measurements and refined simulations are planned for the next beam tests. However, these preliminary TC studies confirm the high quality of chargecollection-and transport properties of the tested samples, as predicted by the spectroscopic measurements (Section 3).

\section{Summary and conclusions}

A characterization method for spectroscopic grade SC-D is described, which simplifies the evaluation of diamond to the measurement of only one parameter: the energy resolution of a sensor made of that material in HI spectroscopy experiments. The key feature of the method is the favourable moderate space-charge limited conditions provided by fully-ionized relativistic HIs. Swift ions create a homogeneous ionization profile through the whole sample thickness and deliver mono-energetic energy-loss lines of a strength and shape easy to detect and simple to interpret.

The preliminary data analysis convincingly shows a way to a quantitative description of carrier-trapping defect concentration limits and to a qualitative characterization of charge transport in the $\mathrm{SCL}$ regime. The unexpected good energy-loss resolution $\delta E_{\mathrm{FWHM}} /$ $\Delta E \sim 2.5 \%$, corresponding to a particle identification power $\Delta Z \mid Z<1$ measured with SC-D samples in the range $41 \leq Z \leq 54$, is in the order of the expected values of a HI spectroscopy detector close to ideal. The study of SCLC transients confirms the spectroscopic results and thus the potential of the characterization method.

\section{Acknowledgments}

This work is supported by the European Community through the Integrated Infrastructure Initiative Hadron Physics, Project RII3-CT2004-506078. We would like to thank the FRS group for perfect support during the preparation and run of the experiment, the ladies of the Target Laboratory for sample metallization, and the GSI accelerator operating for the excellent HI beams. The corresponding author is grateful to W. Koenig for comments to this manuscript and to C. Kozhuharov for many helpful discussions.

\section{References}

[1] J. Lindhard, A.H. Sørensen, Phys. Rev. A 53 (1996) 2443.

[2] A. Many, G. Rakavy, Phys. Rev. 126 (1962) 1980.

[3] G. Juška, M. Viliunas, O. Klíma, E. Šípek, J. Kočka, Phil. Mag. B 69 (1994) 277.

[4] G. Juška, M. Viliunas, K. Arlauskas, J. Kočka, Phys. Rev. B 51 (1994) 66816.

[5] E. Berdermann, K. Blasche, H.W. Daues, P. Moritz, H. Stelzer, B. Voss, Proc. of the 7th Int. Conf. on Advanced Technology and Particle Physics, ICATPP-7, Como Italy, 2001.

[6] P. Bergonzo, F. Foulon, A. Brambilla, D. Tromson, C. Jany, S. Haan, Diam. Rel. Mat. 9 (2000) 1003.

[7] M. Rebisz, B. Voss, A. Heinz, E. Usenko, M. Pomorski, Diam. Rel. Mat. 16 (2007) 1070 .

[8] H. Pernegger, S. Roe, P. Weilhammer, V. Eremin, H. Frais-Koelbl, E. Griesmayer, H. Kagan, S. Schnetzer, R. Stone, W. Trischuk, D. Twitchen, A. Whitehead, J. Appl. Phys. 97 (2005) 073704.

[9] M. Pomorski, E. Berdermann, M. Ciobanu, A. Martemiyanov, P. Moritz, M. Rebisz, B. Marczewska, Phys. Stat. Sol. A 202 (2005) 2199.

[10] M. Pomorski, E. Berdermann, A. Caragheorgheopol, M. Ciobanu, M. Kiš, A. Martemiyanov, C. Nebel, P. Moritz, Phys. Stat. Sol. A 203 (2006) 3152.

[11] J. Isberg, A. Lindblom, A. Tajani, D. Twitchen, Phys. Stat. Sol. A 202 (2005) 2194.

[12] H. Kagan, NIM A 546 (2005) 222.

[13] L. Landau, J. Phys. USSR 8 (1944) 201

[14] C. Scheidenberger, H. Geissel, H.H. Mikkelsen, F. Nickel, S. Czajkowski, H. Folger, H. Irnich, G. Muenzenberg, W. Schwab, T. Stöhlker, T. Suzuki, B. Voss, Phys. Rev. Lett. 77 (1996) 3987.

[15] U. Fano, Annu. Rev. Nucl. Sci. (1963) 1

[16] Element Six Ltd., Ascot, Berkshire, UK.

[17] H. Geissel, H. Weick, C. Scheidenberger, R. Bimbot, D. Gardès, NIM B 195 (2002) 3.

[18] E. Berdermann, M. Pomorski, A. Pullia, S. Riboldi, M. Träger, H. Weick, D. Boutin, H. Geissel, Yu Litvinov, C. Nociforo, K. Sümmerer, M. Winkler, Proc. XLV Intern. Winter Meeting on Nuclear Physics, 2007 available online: http://wspig2.physik.unigiessen.de:8000/Bormio2007/uploads/EleniBerdermann.pdf.

[19] H. Sann, NIM A 392 (1997) 433.

[20] E. Berdermann, K. Blasche, P. Moritz, H. Stelzer, B. Voss, F. Zeytouni, Nucl. Phys. B 78 (1999) 533 (Proc. Suppl.). 